\title{
Publisher Correction: Atomic scale imaging of magnetic circular dichroism by achromatic electron microscopy
}

Zechao Wang (D), Amir H. Tavabi, Lei Jin, Ján Rusz (D), Dmitry Tyutyunnikov, Hanbo Jiang, Yutaka Moritomo (D), Joachim Mayer, Rafal E. Dunin-Borkowski, Rong Yu (D), Jing Zhu (D) and Xiaoyan Zhong

Correction to: Nature Materials https://doi.org/10.1038/s41563-017-0010-4, published online 5 February 2018.

In Fig. 1 of the version of this Letter originally published, the word 'Subtract' was missing from the green box to the left of panel $\mathbf{f}$. This has now been corrected in all versions of the Letter.

Published online: 14 February 2018

https://doi.org/10.1038/s41563-018-0039-z 